\title{
GENDER AND YOU
}

\section{Transforming Cultures eJ ournal, \\ Vol. 2 No 2, December 2007 \\ http:// epress.lib.uts.edu.au/journals/TfC}

\section{Alan Sondheim ${ }^{1}$}

Michael Current and I started Cybermind back in 1994; we wanted a forum for discussion of cyberspace theory and practice, issues of embodiment and sexuality and so on. That's my background.

For me, the most overwhelming arena of writing, in which the body appeared fullest so to speak, was ytalk. With Cybermind and the other lists I send to, the writing is cooler (although it might not appear such). Nowadays my experiences are generally in the form of feedback from writing; the intimacy isn't there, and couldn't be there at this point - it would interfere too much with my private life. In this I'm echoing what Dibbell found in My Tiny Life - I had to move on. Or to some extent, the Net has evolved elsewhere; with the close to perfect emergence of real-time detailed video, for example.

Initially I found myself exploring any number of Internet venues, most of them ascii at that time (what I've called 'darknet' although that word now seems used otherwise); I also started teaching Net matters, practice or theory, etc. One exercise - I asked people to log on to various IRC channels as 'Susie' or some such, no matter what the gender.

\footnotetext{
${ }^{1}$ Alan Sondheim is an artist, philosopher and compulsive writer. Since January, 1994, he has been working on the "Internet Text", a continuous meditation on philosophy, psychology, language, body, and virtuality. He has been artist or writer in residence at many sites, and has taught at Florida International University in Miami and Brown University in Providence, Rhode Island. Currently he is working on a National Science Foundation grant at West Virginia University, researching codework, computer literature, virtual environments, and avatars. His books include the anthology Being on Line: Net Subjectivity (Lusitania, 1996), Disorders of the Real (Station Hill, 1988), .echo (alt-X digital arts, 2001), Vel (Blazevox 2004-5), Sophia (Writers Forum, 2004), and Orders of the Real (Writers Forum, 2005). He has assembled a special issue of the American Book Review (Vol.22, No.6, 2001) on Codework. Records and CDs have been issued by ESP-Disk, Qbico and FireMuseum, and his video and film have been shown internationally.

Relevant URLS:

$<$ http://www.alansondheim.org/>, Blog: <http://nikuko.blogspot.com/>, WVU 2004 projects:

$<$ http://www.as.wvu.edu/clcold/sondheim/files/>, recent WVU:

$<$ http://www.as.wvu.edu:8000/clc/Members/sondheim/>
} 
Most of the time, the screen would immediately light up with bold-face characters private messagings - asking for private contact - clearly for sexual purposes. There was always this air of marauding.

On the other hand, there's the literally sticky issue of (in my case, heterosexual) netsex, which I participated in, and wrote about, for quite a while. At that point, the practice was largely textual, although CuSeeMe was used on occasion; part of the lure of the latter was the slowness and breakup of the image, which created a kind of 'unveiling' through delay, pixellation, etc. Delay also characterized ytalk, a popular software application for net sex - with ytalk, one could see the other person's typing simultaneously with one's own; each participant 'had' a different portion of the screen, which also represented both participatory and interwoven speech, as well as adjacent bodies. (You can see some ytalk dialog at <http://www.asondheim.org/l.txt> by the way.)

I used the term 'jectivity' to refer to the complex of projections and introjections that characterize the imaginary of net sexual communication. Jectivity relates to the flow of the imaginary or imaginary flux, between partners - that goes both ways, that resonates, echoes both ways (when I respond to what I think she is thinking of me, etc.). This is unconscious or rather preconscious (although these terms are outdated). The flow contains voice, image, sound, etc. all in relation to the limited bandwidth available online. These complexes of projections and introjections (and perhaps interjections) when they occur online seem to relate to pre, or non, linguistic phenomena, although they're formed and characterized by language.

I also developed a theory of 'rewrite' - that one is only online, i.e. given ontological status - to the extent that one repeatedly self-inscribes. Rewrite fascinates; it may refer to nothing more than the actions of a Julia bot, for example. Ontology is confused; if one assumes any variety of Turing test here, then rewrite would imply physical embodiment. We take it as such, in the same way that we take a photograph as constituting the evidentary real, even though we know better. Rewrite, unlike a photograph, is more directed; it's process, temporal. 
Around this time, I rewrote the Eliza-doctor script in Emacs, replacing the doctor with Nikuko, who operated harshly in the environment somewhere between seduction and abject distaste. It could fool no one but nonetheless worked; the dialog veered between cartoon and confession.

In terms of gender, one of the things that surprised me, particularly in relation to the media, was the almost total absence of subterfuge; in spite of the notion that one 'can be anyone' online, most people tried to represent themselves accurately. There were not, as far as can be told, large numbers of white heterosexual males masquerading as the 'other'.

I also found that online sexual experience could be inordinately strong with almost violent orgasms (this seemed gender independent); I worked through the notion of the 'ascii unconscious' to represent the letter/shifter in the service of desire. The ascii unconscious relates to Lacan; the idea that the unconscious is structured like a language, which, however, I don't believe. But I do follow Merlin Donald who writes about the literal extensions of mind into the environment vis-a-vis databasing, etc. So the ascii unconsious is part extension, part catalyst, part resonant response to the other, only part of which is consciously deliberated; it forms a partial mapping of desire and partialobjects; it replaces the real, insofar as communication is online. The ascii unconscious is also a very reduced modality of course.

Sexual temporality and spatiality were also much more complex than they might have seemed otherwise. The temprality is constantly an adjudicated, adjusted flow; it's not necessarily forward - it's held back. Space is of the imaginary, even when using CuSeeMe - it's not present, it's inferred. One moves through the other's anatomy.

For years, I wrote through three characters - Jennifer, Julu, and Nikuko - all women. I found I couldn't write through a male character; I feel diminished in this respect, and by writing otherwise, I was able to write through and into desire - Nikuko for example represented a kind of demiurge, a witness to war and destruction, a creator (sexual and otherwise), a magician. This was related to the Sun Goddess in the Kojiki, among other things. I did have male characters as well - Doctor Leopold Konninger, Travis, and Alan - but I felt little or no identification - perhaps their notions of masculine/phallic 
control were displacing my own. I found I could analyze both the experience and theorization of control better from a distance which nonetheless short-circuited. The Nikuko work led to a number of videos and scripts, as well as dance material which was used elsewhere choreographically. At times the characters got the better of me Jennifer in particular attacked me online. At one point I managed to hack into a Jennifer newsgroup and posted as a Jennifer - I wanted to see first, if I could hack into anything, and second, I wanted to create more of a basis for her reality.

The character's reality is partially gained through expectation of 'fluid identity' of the kind discussed by Turkle, even through what seems to be merging and emerging of identities. Ultimately this 'reality' was generated by 'me' rather than just by rewrite, but it also relates to, I think, Lacan's use of 'shifter' to characterize the construct of the ego through language - the 'I' that appears isn't always the same 'I.' But of course it really is the same ' $\mathrm{I}$ ' to the extent that it originates in and references the inert/idiotic (Rosset) real body of the participant, i.e. the person typing/stroking/etc.

Jennifer's reality, such as it is, was only my own, and I'm well aware of the play of both mirrors and mirror-stages (not to mention the apparatus of dream-screens and the uncanny) in this regard. I wanted to explore this play; I also wanted to deal with the luminance of echo/self-referentiality/doubling as, for example, Julu and Jennifer would meet in a talker, or MOO, or on ytalk, or IRC, etc. Nikuko in particular disrupted IRC channels (I had some war programs); the results were instructive. Julu/Jennifer went late Heideggerian. With the disruption, it could lead to a situation where I would start to get into trouble either by being kicked off or by the possibility of dcc stuff coming through. Sometimes Nikuko was taken for a bot, sometimes ignored. The result of all of this is in my Internet Text.

It needs to be stated that this form of writing is not deceit or cruising for sex. In the environments in which I work (except on the Jennifer newsgroup - which lasted maybe two days), everyone knows that these are fictitious creations. I don't disguise myself and never have and have no interest in doing that and for the most part find the idea unethical. I'm not attempting to 'switch genders' - I have essays on the inconceivability of this going back at least to the 80s. Neither am I attempting some kind of drag performance or 'buffoonery' - although some people can confuse the writing with that. 
Furthermore, in netsex (which the work does engage with) identities are mostly transparent, and so here. What it _is_ is an opportunity to try and write through the Heideggerian issues of being, reflection, releasement, etc. that I'm interested in, without bringing along, on the surface, the male baggage that characterizes the analysis of such issues.

Roles are problematic offline as well as online, in political, descriptive or existential terms at least. Say someone were to write they were "a woman writing as a woman” to point out that I am not female yet writing with female avatars. What does this mean? As a Jew, am I always writing as a Jew or only as a Jew? As a Jew, what might it mean to write not as a Jew but as an atheist or as a Christian? Can nothing be said or learned or imagined? Can no writer ever even try to write characters of the other sex, another culture, another race, another species (no animals!). These exclusionary roles seem strangling to me - and they_are_ roles - there is no "a woman" any more than "a male" or “a Jew”. This does not mean that by 'writing as a woman' I gain access to the same knowledge a woman has, as if there were a universal involved. However, something can be learned: for example when a male student logs into IRC as a woman and sees and feels what's happening (no, not like “a woman” but like someone who may be more sensitive to attack) he (or she for that matter) learns a lot more than asking a 'woman' what “it's like” - since 'what it's really like' can imply an essentialism which online experience doesn't. Yet it seems so easy to assume that you (or I) know who I am (or who you are), or what I (or you) have experienced, and the same of them. Roles are like a knowledge which stops us from knowing, because we already 'know'.

Despite this talk, these characters - except for in this, and one other recent, text haven’t appeared in quite a few years. At this point I write through myself.

So, in this writing, there were various modes at work here - representations of vocal communication, play scripts, set pieces, writings by one or another avatar (for that is what I considered them), postings on email lists or usenet groups or webpages, and video/audio tapes as well as live or recorded performance.

When I work with video and/or script or dance or sound or performance, that's live or meant to be presented as products from live performance. It's there that dis/comfort 
emerges; the result is taken as real, whether or not it is. And the modality of performance, dance for example, is wider than one might imagine - you smell the sweat of the dancer, perhaps are physically touched (not often), and the sounds are all sorts just as if one is attending an orchestral performance in which one hears the sounds of the flute pads and bow screeching.

Again, all of this appeared in a swirl of theory; I would log live communication, for example, and analyze it later. Since I was (and am still) working and developing notions of 'codework,' I'd operate with disrupted materials, corrupted theory, abject analyses; this hopefully resulted in a 'readerly' therapeutic, i.e. the reader to some extent working through the content of the material. (If you look at <http://www.asondheim.org/d.txt $>$ or $<$ http://www.asondheim.org/Weather.txt> you'll find some of these texts.)

All of the characters, both female and male, represented the 'wayward' or 'contrary' the bad-girl, bad-boy, the disruption, screwed etiquette; the sleazy or ragged, nightclub or dayclub. So there was a political component as well. There was also energy; these avatars were explorers breaking into new spaces, new lands, or old and shifting territories. I think there was an optimism as well, something which has been lost for me, as the world itself turns increasingly dark and self-destructive. Which is not to deny there was violence in the past of course. Then the avatars fought wars of their own, plays and play-acting; they toyed with each other. Now, child abuse has become a screen for right-wing control of the social, and men, women, and children are routinely destroyed in bombings and pillage. So the analyses I worked on were analyses, in a sense, of peace or peace- time. I write darker now; both violence and reconstitution (of bodies, languages, sexualities) have come more to the foreground. The work, nowadays, is about war, violence, and the bedrock of those aspects of western culture which produce Britney Spears and Iraq, as well as occasionally healthy sexuality. My work for the past 4-5 years has been performance; the texts are put online. There's no gender playing but a lot of analysis. So it goes.

I do want to note here, as I've talked mainly about text, that CuSeeMe was close to another experience altogether. Here the real itself is uncanny, one body maps onto another, seduction and the parasitism of noise merge, display fixed and fetishized the abject, everything gave up, gave in, proof and ‘truth' replaced rewrite, ytalk meetings 
were replaced by assignations, and there was always the possibility of third parties. With ytalk, writing and erasing a line is a seductive form - I said such-and-such, I took it back, but it's present, remains there, under erasure. With CuSeeMe, there's no taking back, there's only continuity, process, delirous negotiation.

In short my experience has taken gender for granted to some extent, and in this sense has been false; I have pushed everywhere (and been pushed) except across the divide of problematizing, and in that sense what I've accomplished is most likely useless here. (And as indicated above, useless because of its production, occurrence, at an historical moment, long since past; just as extinguished species do not return, these moments are gone in a fast-forward world which digests and spews out sexualities at increasingly frenetic rates.) 